\title{
Novel chloroplast microsatellite markers in pigeonpea (Cajanus cajan L. Millsp.) and their transferability to wild Cajanus species
}

\author{
Swati Saxena ${ }^{1}$, Tanvi Kaila ${ }^{1}$, Pavan K. Chaduvula ${ }^{1}$, Archana Singh $^{2}$, N. K. Singh ${ }^{1}$, Kishor Gaikwad* ${ }^{1}$ \\ ${ }^{1}$ ICAR - National Institute for Plant Biotechnology, Pusa Campus, New Delhi- 110012, India \\ ${ }^{2}$ Division of Biochemistry, Indian Agricultural Research Institute, New Delhi- 110012, India
}

*Corresponding author: kish2012@nrcpb.org

\begin{abstract}
Chloroplast microsatellites are potential genetic markers which provide insight into plant systematics studies. A set of 39 new chloroplast microsatellite markers were developed by analyzing the pigeonpea chloroplast genome sequence. Primer pairs were designed for 39 random sequences containing mononucleotides, dinucleotides, tetranucleotides and compound repeat motifs. The newly developed chloroplast SSRs were checked for their transferability in six wild Cajanus species (one accession each). 17 of the 39 cpSSRs displayed polymorphism among the wild Cajanus species with an average polymorphism information content (PIC) value of 0.40 . In total, 41 alleles were produced at the polymorphic loci, each marker generating on an average 2.41 alleles per locus. This point towards the effectiveness of the primer pairs in detecting genetic relatedness amid species within the genus Cajanus. Genetic relationship based on neighbor-joining method revealed two major groups (Group I and group II) of which Group II consisted of two main clusters including the cultivated Cajanus cajan and other wild Cajanus species except for one wild species $C$. platycarpus that was most diverse from rest of the species.
\end{abstract}

Keywords: Cluster analysis; genetic diversity; phylogenetic study; polymorphism; SSRs; wild relatives.

Abbreviations: AFLP_amplification fragment length polymorphism; CMS_cytoplasmic male sterility; cpSSRs_chloroplast simple

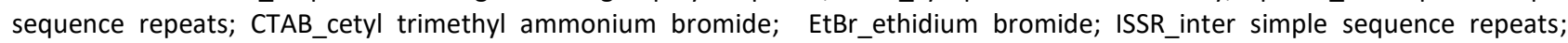
PIC_polymorphism information content; RAPD_randomly amplified polymorphic DNA; RFLP_restriction fragment length polymorphism; SSRs_simple sequence repeats.

\section{Introduction}

Pigeonpea \{Cajanus cajan (L.) Millsp.\} is a vital legume crop which belongs to the family Fabaceae with an estimated 858 Mbp genome size (Greilhuber and Obermayer, 1998) and is often cross-pollinated. Pigeonpea is the second major grain legume crop holding an important position in the rain-fed agriculture (Saxena et al., 2010). India is considered as the center of origin of pigeonpea (Van der Maesen, 1980) which then successfully traveled to East Africa and also to the American continent. The three major pigeonpea producing regions are the Indian sub-continent, central-America and eastern Africa. It is well known as a protein-rich crop with $21 \%$ protein content in the seeds along with the presence of various vital amino acids (Dutta et al., 2013). Pigeonpea is globally cultivated on 5.32 Mha land area with an overall annual production of $4.32 \mathrm{Mt}$ (FAO 2013). The states of Maharashtra, Madhya Pradesh, Gujarat, Uttar Pradesh, Karnataka and Andhra Pradesh account for over $70 \%$ of the total pigeonpea area in India (Saxena and Nadarajan, 2010). In the past five decades, a steady growth in production of pigeonpea has been recorded; however average yield remains static at around $700 \mathrm{~kg} / \mathrm{ha}$ (Nadarajan et al., 2008). Pigeonpea is greatly influenced by different biotic and abiotic factors which restrain its yield capacity. The resilience to all these challenges is absent in the cultivated genotypes but on the other hand, few of the wild relatives offer strong resistance to these constraints (Mallikarjuna et al., 2011). The wild species of pigeonpea possess various desirable attributes such as high protein content, dwarf growth habit and cytoplasmic male sterility (CMS) (Saxena et al., 2002; Saxena and Sharma, 1995; Mallikarjuna and Saxena, 2005; Saxena et al., 2005). Extensive morphological variations exist within cultivated species of pigeonpea but are not available as they display low levels of polymorphism. In contrast, the wild relatives of pigeonpea provide a vital source of genetic variability with respect to resistance to diseases, pest and drought (Panguluri et al., 2006).

Estimation of genetic diversity and its utilization in the breeding programme is of key importance for crop improvement. In pigeonpea, morphological and biochemical (isozymes) markers have been previously employed for the estimation of genetic diversity of the cultivars and the wild species but these markers are influenced by environmental conditions and are not considered as a reliable source for genetic diversity estimation (Ratnaparkhe et al., 1995). In contrast, the desirable attributes of DNA molecular markers such as highly polymorphic, co-dominant inheritance, 
frequent distribution in the genome, high reproducibility, easy availability and neutral to the environmental conditions have made them a versatile tool to measure genetic relationship in crop plants (Joshi et al., 1999). Several DNA markers including RFLP (Nadimpalli et al., 1993), RAPD (Ratnaparkhe et al., 1995), AFLP (Panguluri et al., 2006) and ISSR (Yadav et al., 2014) were successfully employed for estimation of intra-specific and inter-specific genetic diversity and phylogenetic studies in pigeonpea. In past years, SSRs have gained significant importance and are widely accepted class of molecular markers for genetic analysis of crop plants (Oliveira et al., 2006).

Simple sequence repeats (SSRs) or microsatellites are short DNA stretches of single specific loci containing one to six bases which are tandemly repeated (Schlotterer, 2000). Eukaryotic genomes are densely interspersed with simple sequence repeats which exhibit a high degree of polymorphism as a result of length variation due to the occurrence of a different number of repeat units (Morgante and Olivieri, 1993). Microsatellites are preferred DNA markers as these are abundant in the genome, multiallelic nature, highly variable, co-dominant inheritance, reproducibility and amenability to automation and high throughput genotyping (Powell et al., 1996). In similar fashion to nuclear SSRs, chloroplast microsatellites (cpSSRs) also exhibit polymorphism due to length variability as consequences of alterations in the number of repeat motifs (Powell et al., 1995).

Chloroplast simple sequence repeats (cPSSRs) are generally located in the non-coding regions inclusive of introns and intergenic spacers of the cp genome. Chloroplast SSRs show remarkable intra-specific variation and thus are contemplated as efficient markers in the evolutionary and systematic investigation in plants (Provan et al., 2001). Till date, 191 SSRs have been identified in pigeonpea through enrichment of genomic libraries (Burns et al., 2001; Odeny et al., 2007, 2009). With advancement in the sequencing technologies, 3072 SSRs were reported using BAC end sequencing (Bohra et al., 2011). Deep transcriptome studies further identified 3583 and 3771 genic SSRs in pigeonpea (Raju et al., 2010; Dutta et al., 2011). A total of 189,895 genomic SSRs have been added to the previously available SSRs with the release of the pigeonpea draft genome sequence (Singh et al., 2012). From the above studies, a complete set of genic and genomic SSR markers has been developed as a significant genomic tool for diversity assay in pigeonpea. However, till date development of chloroplast SSR markers (cPSSRs) has not been reported in pigeonpea. In the current study, the cpSSRs so developed were used for discerning genetic relationship among Cajanus cajan and six wild Cajanus species.

\section{Results}

\section{Microsatellite mining, development of cPSSR markers and their genetic assessment}

Pigeonpea chloroplast genome sequence (accession number: KU729879) was used for SSR mining (Kaila et al., 2016). A total of 292 chloroplast SSRs (cpSSRs) were mined in pigeonpea using MISA perl script. Primer sets flanking 39 SSR loci were designed and designated as Cajanus cajan chloroplast (Cccp) SSR markers (Table 1). One accession each of wild Cajanus species including C. cajan was utilized for testing cross-species amplification of the newly developed chloroplast SSRs. All the cPSSR markers demonstrated scorable results with $100 \%$ transferability efficiency. 17 of the 39 Cccp SSR markers were polymorphic (43.5\%) and remaining was found to be monomorphic. A PCR amplification profile produced by one of the polymorphic markers is given in Fig. 1. Overall, 41 alleles were generated by the polymorphic loci with an average of 2.41 alleles for each locus. The number of alleles at these loci varied from 2 (Cccp_03, Cccp_04, Cccp_05, Cccp_10, Cccp_11, Cccp_14, Cccp_23, Cccp_28, Cccp_32, Cccp_35, Cccp_37, Cccp_39) to 5 (Cccp_08). The discriminatory competence of each SSR marker was evaluated by PIC values that varied from 0.21 to 0.71 (average $=0.40$ ). Likewise, the major allelic frequency for these polymorphic loci varied from 0.43 (Cccp_08 and Cccp_13) to 0.87 (Cccp_11, Cccp_23, Cccp_28, Cccp_32 and Cccp_39) with average of 0.68 (Table 2). Majority of the polymorphic loci include mononucleotides (47\%), dinucleotides $(29.4 \%)$, one tetranucleotide and three compound repeats.

\section{Phylogenetic study of Cajanus cajan and wild Cajanus species}

The polymorphic cpSSR markers were efficient in differentiating the individual genotypes in the present study. The allelic data generated by them was utilized to compute Nei's genetic distance values and to construct a dendrogram (Fig. 2). The neighbor-joining cluster analysis reported two groups; Group I and Group II with $100 \%$ bootstrap values suggesting complete support to the grouping pattern. Group I, includes only one genotype that was distinctly different from other genotypes forming Cluster I. Group II was divided into two clusters of which Cluster II and Cluster III contained 3 genotypes each. Cluster I represent only single wild species Cajanus platycarpus which was an outlier. Cluster II contained Cajanus lineatus, Cajanus acutifolius and Cajanus sericea (bootstrap values varied from $7 \%$ to $20 \%$ ) on the other hand Cluster III contained Cajanus cajan and wild species Cajanus cajanifolius and Cajanus scarabaeoides (bootstrap values 50\%).

\section{Discussion}

Mining of cPSSR markers and their utilization in genetic assessment

Chloroplast microsatellites (cpSSRs) are extremely useful due to the conserved gene order, uniparental and nonrecombinant inheritance of the chloroplast genome (cpDNA) making these proficient for phylogenetic and evolutionary studies in plants (Olmstead and Palmer, 1994). Previously, a large set of genic and genomic SSRs employed for genetic variability studies in pigeonpea were reported (Burns et al., 2001; Odeny et al., 2007, 2009; Raju et al., 2010; Bohra et al., 2011; Dutta et al., 2011; Singh et al., 2012). The number of cPSSR mined in the present study was higher than the ones identified in Sesamum indicum, Vigna radiata and Camellia species (Yi and Kim, 2012; Lin et al., 2015; Huang et 
Table 1. List of cpSSRs primer pairs used for genetic relationship studies among pigeonpea and its wild relatives.

\begin{tabular}{|c|c|c|c|c|c|c|}
\hline S.No & Primer Id & Repeat motif & Left Primer Sequence 5'-3' & Right Primer Sequence 5'-3' & Allele size/range (bp) & $\mathrm{Tm}^{\circ} \mathrm{C}$ \\
\hline 1 & Cccp_01 & (A)11 & CAGTTTATGCATGGCGAAAG & GCAACAACTGGTACCATAGA & 217 & 52 \\
\hline 2 & Cccp_02 & (A)13 & ACGTGATGATATAGCCCAAC & ACCTGTTCTTTCCATGACTC & $140-200$ & 52 \\
\hline 3 & Cccp_03 & (T)10 & GGGCTCTTTGACTGTAGAAA & ССАТСТСТССССАATTGAAA & $180-231$ & 55 \\
\hline 4 & Cccp 04 & (A) 10 & GATCAAAAGTTTATCCGCGG & CCTTTTCTTGCCCGTATTTC & $245-255$ & 55 \\
\hline 5 & Cccp 05 & (A) 22 & ATGGTATGTTGCTGCCTTTT & CCTTTTCTTGCCCGTATTTTC & $186-195$ & 52 \\
\hline 6 & Cccp_06 & (A)14 & ACGCGTAAATAGATTGACCT & TGGAATTGTCATAGTTTTCGA & 242 & 55 \\
\hline 7 & Cccp_07 & (A) 20 & AGCATAGAAGAAACCCATGT & GAAACCATTCTTTTGAATTCTTCG & $224-330$ & 55 \\
\hline 8 & Ссcp 08 & (T)13 & CTACTTGGGTATTGAGCGTT & TAGATCCATCCTCCGAAAGA & $150-260$ & 55 \\
\hline 9 & Cccp 09 & (T)10 & CCGTATCATCTTGACTTGGT & TTGTCAACTACTCCTATCGG & 217 & 53 \\
\hline 10 & Cccp_10 & (T)12 & ТTTTСАССТССААТССААСА & AGAGTGCTTCAAAATTCAAACT & $215-232$ & 54 \\
\hline 11 & Cccp_11 & (T)16 & GTTCCCCAACTAGAATGTGT & GGTTGTTGTTATCTGCTCCA & $159-170$ & 55 \\
\hline 12 & Cccp_12 & (AT)5 & CCTAGATACCCGCTTTTTTCT & GGCTTTCTATCTTTGGGATT & 205 & 55 \\
\hline 13 & Cccp 13 & (AT) 9 & ATCATGTCATGTCGATTGTG & TTGAAATCTGTTGGGATAGG & $195-230$ & 56 \\
\hline 14 & $\operatorname{Cccp} 14$ & (TA) 5 & CATTTCATAGGGAACCTCAA & TTTTCAACTTTCCATTTTCC & $150-160$ & 54 \\
\hline 15 & Cccp_15 & (AT)5 & TTTTCAACTTTCCATTTTCC & САTTTCATAGGGAACCTCAA & 159 & 54 \\
\hline 16 & Cccp_16 & (CT)5 & CTGGATATCTGTTCCCCATA & TGAAAAATGAAAAGGTCGTC & 173 & 52 \\
\hline 17 & Cccp_17 & (AT)5 & CATATAGATCCCTGCGTTGT & TGGTTCGATTTGAATTATCC & 215 & 55 \\
\hline 18 & Cccp_18 & $($ ATAG)3 & TTCCGAATGGAATAAAAGAG & AAACCCAAATGAACAAAATG & $160-240$ & 53 \\
\hline 19 & Cccp 19 & (A) 12 & CCACATCAAGCACTAACCTC & GCATGTCGTATCAATGAAGA & 242 & 54 \\
\hline 20 & Cccp 20 & (A) 11 & TGTCATTACGTGCGACTATC & AGAAAAAGCGGGTATCTAGG & 246 & 55 \\
\hline 21 & Cccp_21 & (A) 10 & CAGGTTCGAATTCCATAGAG & GCAAGTTGATCGGTTAATTC & 232 & 55 \\
\hline 22 & Cccp_22 & (T)10 & GGTAGAGCACCTCGTTTACA & CATTGACGAGAAATGGGTAT & 224 & 55 \\
\hline 23 & Cccp_23 & (A) 10 & TTTAGGAGATTACCCATTCG & AGCAAAGGGTTTTTCACTTTC & $180-221$ & 55 \\
\hline 24 & Cccp 24 & (T)11 & GATACGTAAGCAAGGCATTT & АTTTTTCTTCGGAGAGTTCC & 224 & 53 \\
\hline 25 & Cccp_25 & (G) 10 & AGGGCAATCACTCATTCTTAA & TCCAAAGAGCAACTCTTCTC & 192 & 54 \\
\hline 26 & Cccp_26 & (A)14 & AACTACTGGAGGGAAAAAGG & AACTGTTTTAACGCCTTTGAG & 236 & 51 \\
\hline 27 & Cccp_27 & (C) 10 & TCCAAAGAGCAACTCTTCTC & AGGGCAATCACTCATTCTTA & 192 & 55 \\
\hline 28 & Cccp_28 & (TA)5 & GACCTTCCAAAATCCTTCTT & GGTTTTTCGTCAATTCCAG & $226-280$ & 57 \\
\hline 29 & Ссcр 29 & (AT) 5 & GCATCTTGAAAGTGAATCGT & GGCTTCTATTGAATCGAGAA & 239 & 52 \\
\hline 30 & $\operatorname{Cccp} 30$ & (AG)5 & AGCTTAACACCTCTCATTCCT & CTGGATATCTGTTCCCCATA & 226 & 53 \\
\hline 31 & Cccp_31 & (TA)6 & ATACACCCTGGTACACGTTC & AAGTAATTCGGGGTTAGGAT & 183 & 54 \\
\hline 32 & Cccp_32 & (АT)6 & CTGCCGTTTTTATAGTTTCC & TATCAAGAGACCTGCCAAAG & $236-270$ & 52 \\
\hline 33 & Cccp_33 & (A) $8 \mathrm{~N}(\mathrm{AT}) 5 \mathrm{~N}(\mathrm{AAAT}) 3$ & TTGAAAACCGGTATAGTTCG & TGAAAAAGGAATTGATCGAG & 318 & 56 \\
\hline 34 & Cccp_34 & (AT) $5 \mathrm{~N}(\mathrm{TA}) 6$ & AGGCCTTATCCACACAAGTA & GCGGGTATCTAGGCATATCT & 351 & 56 \\
\hline 35 & Cccp 35 & (TTA) $5 \mathrm{~N}(\mathrm{ATT}) 5 \mathrm{~N}(\mathrm{~A}) 8$ & TAAACCAATTTGCGTCCA & AGAATTATAGAATGAATCCAAA & $310-325$ & 55 \\
\hline \multirow[t]{2}{*}{36} & Cccp_36 & $(\mathrm{T}) 16 \mathrm{~N}(\mathrm{~A}) 8$ & TGATGAATCTTCCATTTTCA & TCAATGACCGAGAATTGTAA & 321 & 53 \\
\hline & בר & (T)13N(A)1 & & 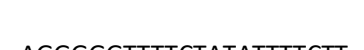 & 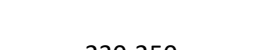 & 55 \\
\hline$\frac{31}{38}$ & $\begin{array}{l}\text { Lccp_3l } \\
\operatorname{Cccp} 38\end{array}$ & 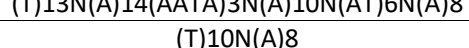 & $\begin{array}{c}\text { TGAAAAATTAATATGGATTACTG } \\
\text { AATGGTTGTTTCTCCCAAG }\end{array}$ & $\begin{array}{l}\text { AGGGGGTTTTCTATATITTCTT } \\
\text { TGGTGTTTCTAACCATCCAT }\end{array}$ & $\frac{230-250}{183}$ & 54 \\
\hline 39 & Cccp 39 & (T)9N(TTTA)3 & CTCAACCTATTTGAATTTTGG & TTTTATCGGACGGTTCTAAA & $130-140$ & 52 \\
\hline
\end{tabular}




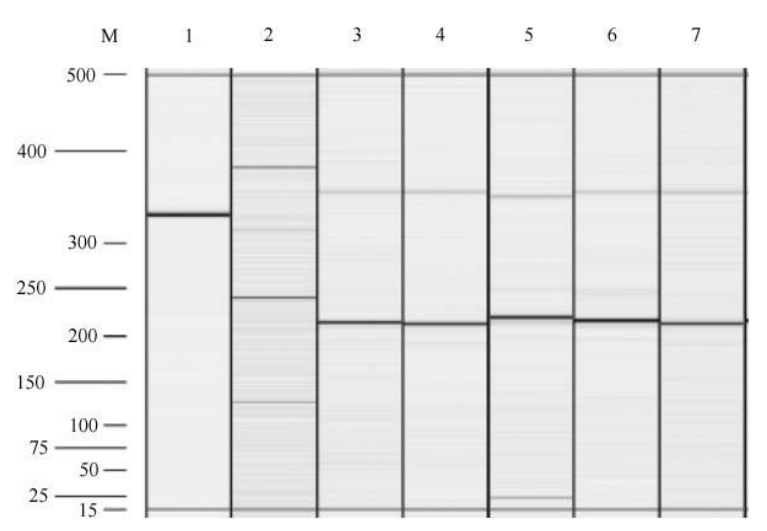

Fig 1. PCR amplification profile generated by Cccp_08 SSR marker among six wild Cajanus species and Cajanus cajan. Lane M- 25-500 bp QX DNA size marker; Lane 1- C. acutifolius; Lane 2- C.cajanifolius; Lane 3- C. lineatus; Lane 4- C.platycarpus; Lane 5- C.scarabaeoides; Lane 6- C.sericea; Lane 7- C.cajan

Table 2. Polymorphism among the six wild and one cultivated Cajanus species.

\begin{tabular}{|c|c|c|c|c|}
\hline S.No & Marker Name & Number of alleles $\left(\mathrm{N}_{\mathrm{a}}\right)$ & Major allele frequency & PIC value \\
\hline 1 & Cccp_02 & 3 & 0.75 & 0.4 \\
\hline 2 & Cccp_03 & 2 & 0.75 & 0.37 \\
\hline 3 & Cccp_04 & 2 & 0.5 & 0.5 \\
\hline 4 & Cccp_05 & 2 & 0.5 & 0.5 \\
\hline 5 & Cccp_07 & 3 & 0.62 & 0.53 \\
\hline 6 & Cccp_08 & 5 & 0.43 & 0.71 \\
\hline 7 & Cccp_10 & 2 & 0.75 & 0.37 \\
\hline 8 & Cccp_11 & 2 & 0.87 & 0.21 \\
\hline 9 & Cccp_13 & 3 & 0.43 & 0.63 \\
\hline 10 & Cccp_14 & 2 & 0.62 & 0.46 \\
\hline 11 & Cccp_18 & 3 & 0.56 & 0.58 \\
\hline 12 & Cccp_23 & 2 & 0.87 & 0.21 \\
\hline 13 & Cccp_28 & 2 & 0.87 & 0.21 \\
\hline 14 & Cccp_32 & 2 & 0.87 & 0.21 \\
\hline 15 & Cccp_35 & 2 & 0.75 & 0.37 \\
\hline 16 & Cccp_37 & 2 & 0.62 & 0.46 \\
\hline \multirow[t]{2}{*}{17} & Ссcр 39 & 2 & 0.87 & 0.21 \\
\hline & Mean & 2.41 & 0.68 & 0.4 \\
\hline
\end{tabular}

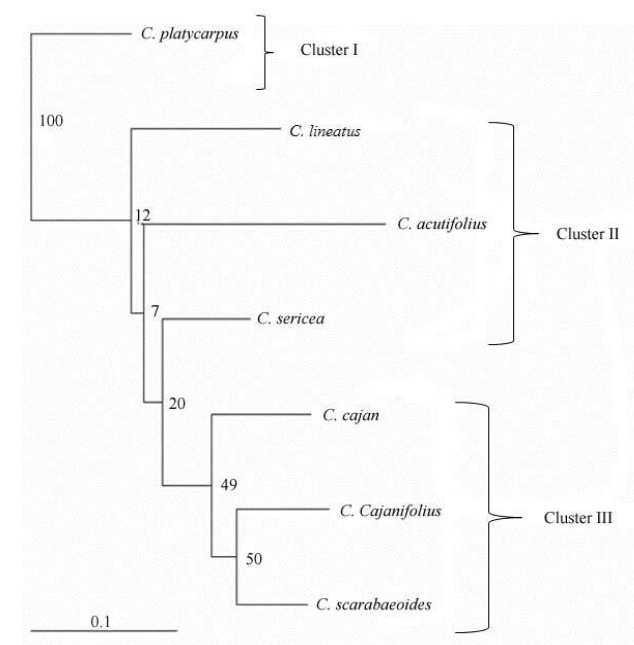

Fig 2. Neighbor-joining tree of six wild Cajanus species and Cajanus cajan obtained by 39 chloroplast SSR markers. 
Table 3. List of wild Cajanus species used for genetic relationship study.

\begin{tabular}{lllll}
\hline S. No & CMS System & Species & Accession ID & Source \\
\hline 1. & A1 & Cajanus sericeus & ICPW160 & ICRISAT, Patancheru, India \\
2. & A2 & Cajanus scarabaeoides & ICPW87 & ICRISAT, Patancheru, India \\
3. & A4 & Cajanus cajanifolius & ICPW28 & ICRISAT, Patancheru, India \\
4. & A5 & Cajanus acutifolius & ICPW1 & ICRISAT, Patancheru, India \\
5. & A6 & Cajanus lineatus & ICPW44 & ICRISAT, Patancheru, India \\
6. & A7 & Cajanus platycarpus & ICPW68 & ICRISAT, Patancheru, India \\
\hline
\end{tabular}

al., 2014). However, the number of SSRs detected in our study was lower in contrast with cpSSRs reported in Glycine species. This could be due to the fact that eight Glycine species were analyzed for SSR mining. The findings were similar to those reported in Glycine max (Ozyigit et al., 2015).

In this study, all the newly developed cpSSRs amplified efficiently and at least one allele was detected in all the accessions indicating their transferability among the wild Cajanus species. The amplification efficiency (100\%) was higher than those reported by Dutta et al. (2011) and Singh et al. (2012). 43.5\% polymorphism was detected by these markers which were higher to $12.9 \%$ for genic SSRs, $28.40 \%$ for genomic SSRs developed via BAC end sequencing and $40.8 \%$ for genomic SSRs previously used for genetic diversity assessment in Cajanus cajan (Dutta et al., 2011; Bohra et al., 2011; Singh et al., 2012). This point towards the high and efficient utility of these cPSSR markers in diversity assessment in this crop. The allelic difference observed at the cPSSR loci varies from 2 to 5 alleles with an average of 2.41 alleles per locus. The findings were comparable to previous diversity analysis studies utilizing SSR markers in pigeonpea (Odeny et al., 2009; Bohra et al., 2011; Mudaraddi et al., 2013; Petchiammal et al., 2015). The mean PIC value of 0.40 was demonstrated by these polymorphic markers which were lower to previously reported genic and genomic SSRs in pigeonpea (Dutta et al., 2011; Mudaraddi et al., 2013). The possible reasons could be the utilization of only highly polymorphic SSRs for genetic relationship studies in the previous reports. The PIC value (0.40) in this study was in accordance with the one documented by Odeny et al. $(2007,2009)$ and higher to ones reported by Petchiammal et al. (2015). These newly developed cpSSRs are therefore competent in genetic diversity, evolutionary and phylogenetic studies in pigeonpea.

\section{Phylogenetic analysis}

The genetic relatedness among Cajanus cajan and six wild Cajanus relatives was established using neighbor-joining clustering which revealed two distinct groups: Group I and Group II (Fig. 2). Group I, consisted Cluster I which included only single wild species Cajanus platycarpus which was an outlier. Cajanus platycarpus was grouped in the tertiary gene pool reason being its low crossability with Cajanus cajan (Dutta et al., 2011). This was evident in our study, as Cajanus platycarpus was completely distinct from the Cajanus cajan and other wild species. Three wild species, namely $C$. lineatus, C.acutifolius and $C$. sericea grouped together in Cluster II. These wild relatives belong to the secondary gene pool and were grouped together in previous reports (Odeny et al., 2007; Dutta et al., 2011). In the study, C. cajanifolius showed close relatedness to $C$. cajan and this was supported by the previous study using genomic SSRS
(Odeny et al., 2007). C. cajanifolius and C. scarabaeoides corresponding to the secondary gene pool grouped together in single cluster III, inferring that these two might have common ancestry (Khera et al., 2015). In this study, C. platycarpus showed close relatedness to $C$. lineatus and $C$. acutifolius which belong to the secondary gene pool. The finding was similar to the one reported by Mudaraddi et al. (2013) where despite being in the tertiary gene pool genetic variation of $C$. platycarpus was similar with $C$. scarabaeoides and $C$. acutifolius and was grouped together.

These novel cPSSR markers could be used as valuable tools to study diversity analysis and can offer new insights into the origin and phylogenetic relationship within the Cajanus genus and other legumes.

\section{Materials and Methods}

\section{Plant materials and DNA isolation}

One accession each of Cajanus cajan (AKPR375) and six wild Cajanus species was used in the present study (Table 3 ). Seeds of wild species were procured from the International Crops Research Institute for Semi-Arid Tropics (ICRISAT), Patancheru, India and the plants were maintained in the research farm at Indian Agricultural Research Institute, New Delhi, India. Leaf samples were collected and frozen in liquid nitrogen and stored at $-80^{\circ} \mathrm{C}$ for further use. Genomic DNA was isolated following a modified CTAB method (SaghaiMahroof et al., 1984). The DNA was analyzed on $0.8 \%(\mathrm{w} / \mathrm{v})$ agarose gel and visualized by EtBr staining. The DNA concentration was measured by Nanodrop spectrophotometer (Thermo scientific).

\section{cPSSR mining and primer development}

High-quality chloroplast DNA sequence of Cajanus cajan (accession number: KU729879) from GenBank (http://www.ncbi.nlm.nih.gov) (Kaila et al., 2016) was employed for detecting chloroplast microsatellites (cPSSRs) using MISA perl script (MIcroSAtellite, http://pgrc.ipkgatersleben.de/misa/) (Thiel et al., 2003). We identified cpSSRs as mononucleotide repeats $\geq 8$ bases, dinucleotides (five repeats), trinucleotides (four repeats), tetranucleotides (three repeats), pentanucleotide (three repeats) and hexanucleotides (three repeats) respectively. Randomly 39 identified sequences containing microsatellite motifs: mononucleotides (20), dinucleotides (11), tetranucleotides (1) and compound SSRs (7) were used for primer designing using Primer3 plus software (http://www.bioinformatics.nl/cgi-

bin/primer3plus/primer3plus.cgi) (Untergrasser et al., 2012). Following parameters were used for primer designing: annealing temperature of the primer between $50-60{ }^{\circ} \mathrm{C}$, 
primer length ( $20-22$ bases), $\mathrm{G}+\mathrm{C}$ content $\sim 40-50 \%$ and the amplicon size varying between $150-350 \mathrm{bp}$. (Table 1 ).

\section{CPSSR amplification and validation}

To test the efficiency of the newly developed cpSSRs, these were employed in genetic relationship studies among one accession each of Cajanus cajan and wild Cajanus relatives following six divergent CMS system: A1 (Cajanus sericeus), A2 (Cajanus scarabaeoides), A4 (Cajanus cajanifolius), A5 (Cajanus acutifolius), A6 (Cajanus lineatus) and A7 (Cajanus platycarpus). Total $20 \mu \mathrm{l}$ reaction mixture comprised of genomic DNA ( $50 \mathrm{ng}$ ), $1 \times$ Taq buffer, $2 \mathrm{mM} \mathrm{MgCl}, 0.25 \mathrm{mM}$ dNTPs, $0.15 \mu \mathrm{M}$ of each primer, and $0.5 \mathrm{U}$ Taq DNA polymerase (NEB). PCR amplification was performed in a thermocycler (Biorad) with an initial denaturation at $95{ }^{\circ} \mathrm{C}$ for $3 \mathrm{~min}, 35$ cycles of denaturation at $95{ }^{\circ} \mathrm{C}$ for $15 \mathrm{~s}$, annealing for $20 \mathrm{~s}$ (temperature for each primer mentioned in Table 1) and extension at $72{ }^{\circ} \mathrm{C}$ for $20 \mathrm{~s}$ followed by final extension at $72{ }^{\circ} \mathrm{C}$ for $5 \mathrm{~min}$. The PCR amplified products were analyzed by capillary electrophoresis using QIAxcel electrophoresis system (QIAGEN). $1 \mu \mathrm{l}$ of amplified PCR product was separated on QX DNA High-resolution cartridge along with QX DNA Size marker 25-500 bp v2.0 for sizing of the amplicons and QX DNA Alignment marker $15 \mathrm{bp} / 1 \mathrm{~kb}$ were run simultaneously. Amplicon sizing was performed with the help of QIAxcel ScreenGel software (QIAGEN).

\section{Phylogenetic study}

To examine the phylogenetic correlation amongst the genotypes included in the study, allelic data were scored as present (1) or absent (0). The Nei's genetic distance values were calculated using Free Tree software version 0.9.1.50 (Pavlieek et al., 1999) and the matrix obtained was used for the construction of the neighbor-joining tree. The bootstrap analyses (based on 1000 re-samplings) of the data were performed to evaluate the authenticity of the clustering pattern. Other parameters including major allele frequency and allele numbers were estimated using POPGENE version 1.3 (Yeh and Boyle, 1999). The polymorphic information content (PIC) was derived by using the formula, $\mathrm{PIC}=1-\sum$ pi 2 (Powell et al., 1996), where pi refers to the frequency of the ith allele.

\section{Conclusion}

A novel set of chloroplast microsatellite markers (cpSSRs) were reported for the first time in pigeonpea. These markers were successfully transferable to six wild Cajanus species with the amplification efficiency of $100 \%$. These cpSSRs markers revealed $43.5 \%$ polymorphism among pigeonpea and its wild relatives depicting their potential in genetic diversity and phylogenetic studies. These newly developed markers will be beneficial in providing information about evolutionary and phylogenetic relationship within the Cajanus genus and other legumes.

\section{Acknowledgements}

The author's thank for the financial assistance received from ICAR- National Research Centre on Plant Biotechnology. Pusa Campus, New Delhi- 110012, India

\section{References}

Bohra A, Dubey A, Saxena RK, Varma Penmetsa R, Poornima $K N$, Kumar N, Farmer AD, Srivani G, Upadhyaya HD, Gothalwal R, Ramesh S, Singh D, Saxena K, Kishor PBK, Singh NK, Town CD, May GD, Cook DR, Varshney RK (2011) Analysis of BAC-end sequences (BESs) and development of BES-SSR markers for genetic mapping and hybrid purity assessment in pigeonpea (Cajanus spp). BMC Plant Biol. 11:56.

Burns MJ, Edwards KJ, Newbury HJ, Ford-Lloyd BV, Baggott CD (2001) Development of simple sequence repeat (SSR) markers for the assessment of gene flow and genetic diversity in pigeonpea (Cajanus cajan). Mol Ecol Notes. 1:283-285.

Dutta S, Kumawat G, Singh BP, Gupta DK, Singh S, Dogra V, Gaikwad K, Sharma TR, Raje RS, Bandhopadhya TK, Datta $S$, Singh MN, Bashasab F, Kulwal P, Wanjari KB, K Varshney $R$, Cook DR, Singh NK (2011) Development of genic-SSR markers by deep transcriptome sequencing in pigeonpea (Cajanus cajan (L.) Millspaugh). BMC Plant Biol. 11:17.

Dutta S, Mahato AK, Sharma P, Raje RS, Sharma TR, Singh NK (2013) Highly variable 'Arhar' simple sequence repeat markers for molecular diversity and phylogenetic studies in pigeonpea (Cajanus cajan (L.) Millisp.). Plant Breeding. 132:191-196.

Food and Agricultural Organization of the United Nation, FAO statistical database (2013) http://faostat3.fao.org/ faostatgateway/go/to/home/E.

Greilhuber J, Obermayer R (1998) Genome size variation in Cajanus cajan (Fabaceae): a reconsideration. Plant Syst Evol. 212:135-141.

Huang H, Shi C, Liu Y, Mao SY, Gao LZ (2014) Thirteen Camellia chloroplast genome sequences determined by highthroughput sequencing: genome structure and phylogenetic relationships. BMC Evol Bio. 14:151.

Joshi SP, Ranjekar PK, Gupta VS (1999) Molecular markers in plant genome analysis. Curr Sci. 77:230-240.

Kaila T, Chaduvla PK, Saxena S, Bahadur K, Gahukar SJ, Chaudhury A, Sharma TR, Singh NK, Gaikwad K (2016) Chloroplast genome sequence of pigeonpea (Cajanus cajan (L.) Millspaugh) and Cajanus scarabaeoides (L.) Thouars: genome organization and comparison with other legumes. Front Plant Sci. 7:1847.

Khera P, Saxena R, Sameerkuma CV, Saxena KB, Varshney RK (2015) Mitochondrial SSRs and their utility in distinguishing wild species, CMS lines and maintainer lines in pigeonpea (Cajanus cajan L.). Euphytica. 206:737-746.

Lin CP, Ko CY, Kuo Cl, Liu MS, Schafleitner R, Chen LFO (2015) Transcriptional slippage and RNA editing increase the diversity of transcripts in chloroplasts: Insight from deep sequencing of Vigna radiata genome and transcriptome. PLoS One. 10:e0129396.

Mallikarjuna N, Saxena KB (2005) A new cytoplasmic malesterility system derived from cultivated pigeonpea cytoplasm. Euphytica. 142:143-148.

Mallikarjuna N, Saxena KB, Jadhav DR (2011) Cajanus. In: Kole $C$ (ed) Wild crop relatives: Genomic and breeding resources, legume crops and forages. Springer-Verlag Berlin Heidelberg, pp. 21-33.

Morgante M, Olivieri AM (1993) PCR-amplified microsatellites as markers in plant genetics. The Plant J. 1:175-182. 
Mudaraddi B, Saxena KB, Saxena RK, Varshney RK (2013) Molecular diversity among wild relatives of Cajanus cajan (L.) Millsp. Afr J Biotechnol. 12:3797-3801.

Nadarajan N, Ganesh Ram S, Petchiammal KI (2008) Fertility restoration studies in short duration redgram (Cajanus cajan (L.) mill spp.) hybrids involving CGMS system. Madras Agric J. 95:320-327.

Nadimpalli BG, Jarret RL, Pathak SC, Kochert G (1993) Phylogenetic relationships of the pigeonpea (Cajanus cajan) based on nuclear restriction fragment length polymorphism. Genome. 36:216-223.

Odeny DA, Jayashree B, Ferguson M, Hoisington D, Crouch J, Gebhardt C (2007) Development characterization and utilization of microsatellite markers in pigeonpea (Cajanus cajan (L.) Millsp). Plant Breeding. 126:130-137.

Odeny DA, Jayashree B, Gebhardt C, Crouch J (2009) New microsatellite markers for pigeonpea (Cajanus cajan (L.) millsp.). BMC Res Notes. 2:35.

Oliveira EJ, Padua, JG, Zucchi MI, Vencovsky R, Vieira MLC (2006) Origin, evolution and genome distribution of microsatellites. Genet Mol Bio. 29:294-307.

Olmstead RG, Plamer JD (1994) Chloroplast DNA systematics: A review of methods and data analysis. Am J Bot. 81:12051224.

Ozyigit II, Dogan I, Filiz E (2015) In silico analysis of simple sequence repeats (SSRs) in chloroplast genomes of Glycine species. Plant Omics J. 8:24-29.

Panguluri SK, Janaiah K, Govil JN, Kumar PA, Sharma PC (2006) AFLP fingerprinting in pigeonpea (Cajanus cajan (L.) Millsp.) and its wild relatives. Genet Resour Crop Evol. 53:523-531.

Pavlieek A, Hrda S, Flegr J (1999) FreeTree- Freeware program for construction of phylogenetic trees on the basis of distance data and bootstrap/jackknife analysis of the tree robustness. Application in the RAPD analysis of Genus Frenkelia. Fol Bio (Praha). 45:97-99.

Petchiammal KI, Muthiah AR, Jayamani P (2015) Molecular characterization of cultivated and wild Cajanus species using simple sequence repeat markers. Legum Res. 38:742-747.

Powell W, Machray GC, Provan J (1996) Polymorphism revealed by simple sequence repeats. Trends Plant Sci. 1:215-221.

Powell W, Morgante M, McDevitt R, Vendramin GG, Rafalski JA (1995) Polymorphic simple sequence repeat regions in chloroplast genomes: applications to the population genetics of pines. P Natl Acad Sci. 92:7759-7763.

Provan J, Powell W, Hollingsworth PM (2001) Chloroplast microsatellites: new tools for studies in plant ecology and evolution. Trends Ecol Evol. 16:142-147.

Raju NL, Gnanesh BN, Lekha P, Jayashree B, Pande S, Hiremath PJ, Byregowda M, Singh NK, Varshney RK (2010) The first set of EST resource for gene discovery and marker development in pigeonpea (Cajanus cajan L.). BMC Plant Biol. 10:45.

Ratnaparkhe MB, Gupta VS, Ven Murthy MR, Ranjekar PK (1995) Genetic finger printing of pigeonpea (Cajanus cajan (L.) Millsp.) and its wild relatives using RAPD markers. Theor Appl Genet. 91:893-898.

Saghai-Maroof MA, Soliman KM, Jorgenson R, Allard RW (1984) Ribosomal DNA spacer length polymorphism in barley: Mendelian inheritance, chromosomal locations and population dynamics. Proc Natl Acad Sci. 81:8014-8018.

Saxena KB, Kumar RV, Rao PV (2002) Pigeonpea nutrition and its improvement. J Crop Prod. 5:227-260.
Saxena KB, Kumar RV, Srivastava N, Shiying B (2005) A cytoplasmic-nuclear male-sterility system derived from a cross between Cajanus cajanifolius and Cajanus cajan. Euphytica. 145:289-294.

Saxena KB, Nadarajan N (2010) Prospects of pigeonpea hybrids in Indian agriculture. Elect J Plant Breed. 1:1107-1117.

Saxena KB, Sharma D (1995) Sources of dwarfism in pigeonpea. Indian J Pulse Res. 8:1-6.

Saxena KB, Sultana R, Mallikarjuna N, Saxena RK, Kumar RV, Sawargaonkar SL, Varshney RK (2010) Male-sterility systems in pigeonpea and their role in enhancing yield. Plant Breeding. 129:125-134.

Schlotterer C (2000) Evolutionary dynamics of microsatellite DNA. Chromosoma. 109:365-371.

Singh NK, Gupta DK, Jayaswal PK, Mahato AK, Dutta S, Singh S, Bhutani S, Dogra V, Singh BP, Kumawat G, Pal JK, Pandit A, Singh A, Rawal H, Kumar A, Prashat GR, Khare A, Yadav R, Raje RS, Singh MN, Datta S, Fakrudin B, Wanjari KB, Kansal R, Dash PK, Jain PK, Bhattacharya R, Gaikwad K, Mohapatra T, Srinivasan R, Sharma TR (2012) The first draft of the pigeonpea genome sequence. J Plant Biochem Biot. 21:98112.

Thiel T, Michalek W, Varshney R, Graner A (2003) Exploiting EST databases for the development and characterization of gene-derived SSR-markers in barley (Hordeum vulgare L.). Theor Appl Genet. 106:411-422.

Untergrasser A, Cutcutache I, Koressaar T, Ye J, Faircloth BC, Remm M, Rozen SG (2012) Primer3-new capabilities and interfaces. Nucleic Acids Res. 40:e115.

Van der Maesen LJG (1980) India is the native home of pigeonpea. In: Libergratulatorius in honorem 9HCD (de Wit Arends JC, Boelema G, de Groot CT, Leeuwenberg AJM eds.) Agricultural University Miscellaneous paper, 19, Wageningen, Netherlands, pp:257-262.

Yadav K, Yadav SK, Yadav A, Pandey VP, Dwivedi UN (2014) Comparative analysis of genetic diversity among cultivated pigeonpea (Cajanus cajan (L) Millsp.) and its wild relatives (C. albicans and C. lineatus) using randomly amplified polymorphic DNA (RAPD) and inter simple sequence repeat (ISSR) fingerprinting. Am J Plant Sci. 5:1665-1678.

Yeh FC, Boyle T (1999) POPGENE version 1.3.2: Microsoft window-based freeware for population genetic analysis. http://www.ualberta.ca/ fyeh/index.htm.

Yi DK, Kim KJ (2012) Complete chloroplast genome sequences of important oilseed crop Sesamum indicum L. PLOS One 7:e35872. 